by Barshes and colleagues, who identified 11 patients from the United Network for Organ Sharing, Organ Procurement and Transplantation Network database, all of whom had end-stage lung and liver disease secondary to cystic fibrosis. Complete follow-up for all patients was available, and survival at 30 days, 1 year, 3 years, and 5 years was $79 \%, 79 \%$, $63 \%$, and $63 \%$, respectively.

The authors also reviewed the Englishlanguage literature available for combined lung and liver transplantation, and identified a detailed report of 9 patients from the UK. The survival curves of the US and UK experiences were similar $(P=0.37)$. The patient survival rates of the US experience of lung-liver transplantation were also comparable with that of isolated orthotopic liver transplantation and isolated lung transplantation rates in the US.

Barshes and colleagues conclude that the favorable outcomes highlighted by the US and UK experiences suggest that combined lung and liver transplantation should be indicated for patients with end-stage liver and lung disease, for whom single-organ transplantation has a poor prognosis.

Katy Cherry

Original article Barshes NR et al. (2005) Combined lung and liver transplantation: the United States experience. Transplantation 80: 1161-1167

\section{Downstaging of hepatocellular carcinoma before transplantation}

The rising incidence of hepatocellular carcinoma (HCC), largely caused by the increasing prevalence of hepatitis $\mathrm{C}$, has fed demand for orthotopic liver transplantation (OLT). Under the Model for End-Stage Liver Disease scheme in the US, only patients with stage T2 $\mathrm{HCC}$ are eligible for priority listing for OLT. Yao et al. have conducted a prospective study to assess the feasibility of downstaging HCC of stage $>$ T2 to bring patients within accepted criteria for OLT.

Patients recruited to the study had one lesion $>5 \mathrm{~cm}$ in diameter, two or three lesions of which one had to be $>3 \mathrm{~cm}$, or four or five nodules all $\leq 3 \mathrm{~cm}$. In all cases the total tumor diameter was $\leq 8 \mathrm{~cm}$. Depending on tumor size and location, the treatments employed were radiofrequency ablation, transarterial chemoembolization, percutaneous alcohol injection, or resection. Downstaging was successful in 21 of 30 patients. Seven patients dropped out because of tumor progression, and two others died of multiorgan failure shortly after treatment. OLT was later performed in 16 of 21 patients; another 4 were awaiting OLT and 1 was excluded for psychosocial reasons. By a median follow-up of 16 months after transplant, 15 patients were alive and 1 had died after 14 months because of poor graft function. The survival rates were $89.3 \%$ after 1 year and $81.8 \%$ after 2 years.

The authors conclude that, while the results are encouraging, expanded studies with extended follow-up are required for full feasibility assessment of downstaging as a means of qualifying patients for OLT.

\section{Jim Casey}

Original article Yao FY et al. (2005) A prospective study on downstaging of hepatocellular carcinoma prior to liver transplantation. Liver Transpl 11: 1505-1514

\section{Distensibility of the gastroesophageal junction is reduced after laparoscopic Nissen fundoplication}

Gastroesophageal reflux disease (GERD) is commonly treated using laparoscopic Nissen fundoplication (LNF), which results in dysphagia in approximately $5-10 \%$ of patients. Blom et al. hypothesized that dysphagia and other complications of LNF, which often discourage physicians from recommending the procedure, might be caused by a reduction in gastroesophageal-junction distensibility (GEJD); therefore, they aimed to describe the effect of LNF on GEJD.

In 15 patients undergoing LNF for GERD, presurgical and postsurgical GEJD were measured intraoperatively using a distensibility catheter. GEJD was also measured after abolishing tone in the lower esophageal sphincter, achieved by inflating a mid-esophageal balloon. Distensibility was measured as both the slope of the pressure-volume curve and the relationship between incremental changes in the pressure and volume of air within the bag.

GEJD was significantly reduced after surgery, with an increase in both the change in pressure caused by each $5 \mathrm{ml}$ increase in bag volume $(P=0.007)$ and in the slope of the pressurevolume curve for volumes between $5 \mathrm{ml}$ and 